\title{
The developmental status and prevalence of sensory integration difficulties in premature infants in a tertiary hospital in Bloemfontein, South Africa
}

\author{
Elise R Lecuona, BOT (UFS), MOT (UFS)
}

Chief Occupational Therapist, Universitas Academic Hospital, Bloemfontein

Annamarie van Jaarsveld, BOT (UFS), MOT (UFS)

Lecturer Occupational Therapy, Faculty of Health Sciences, University of the Free State

Rita van Heerden, BOT (UFS), MOT (UFS), PhD (HPE) (UFS)

Lecturer Occupational Therapy, Faculty of Health Sciences, University of the Free State

Jacques Raubenheimer, BA (UFS), BA Hons (Psychology) (UFS), B.Th (UFS), M.Th. (Practical Theology) (UP), M.A. (Reseach Psychology) (UFS), PhD (Research Psychology) (UFS)

Lecturer Biostatistics, Faculty of Health Sciences, University of the Free State.

Introduction and aim: Research indicates that premature infants are at risk of neurological abnormalities and developmental and functional delays during infancy and early childhood. Annually, in South Africa, approximately 15\% of infants are born prematurely, the majority being from low socio-economic homes. Basic needs and survival of the infant take priority over developmental progress of infants. Since developmental progress is dependent on sensory integration, the aim of this study was to determine the occurrence of developmental and sensory integration difficulties in premature infants in South Africa.

Methods: A descriptive, observational study was conducted. Relevant information on medical history and environmental factors were obtained through parent questionnaires. Three standardised assessments, the Bayley III Scales of Infant and Toddler Development, the Test of Sensory Function in Infants and the Infant/Toddler Sensory Profile, were used.

Results: Infants presented with low average to average performance in all developmental subtests. The majority (67.7\%) of infants presented with typical sensory seeking behaviour. Sensory processing difficulties were identified in terms of high neurological thresholds resulting in low registration behaviour as well as low neurological thresholds, resulting in sensory sensitivity and sensory avoiding behaviour. This influenced their adaptive motor functions and normal development.

Conclusion: Premature infants participating in this research presented with challenges regarding developmental and sensory integration.

Key words: sensory integration difficulties; development; premature infants; South Africa

\section{INTRODUCTION}

Babies in Africa are at high risk of being born prematurely due to factors such as infections, particularly sexually transmitted infections (STIs), HIV/AIDS and malaria'. The premature infant, abruptly removed from the protection of the womb, may experience specific challenges for survival and development since immature organs, especially the brain and lungs, still need to continue their development $^{2}$. An immature nervous system is not ready to process all the sensory information that it is bombarded with in the neonatal intensive care unit (NICU) such as, bright lights, loud noises, an intrusive environment, painful interventions, and unintentional and repeated disruption of sleep ${ }^{2}$, these infants are at increased risk for sensory integration (SI) problems ${ }^{3}$.

More than 8 out of 100 babies are born prematurely in South Africa per annum ${ }^{4}$. These babies' growth and development postdischarge from the NICU is further affected by factors such as parents' socio-economic level, culture, educational level, health history and home environment. For low socio-economic families, basic needs, health and provision of food remain a priority. Home environments may be small with limited resources and a lack of knowledge of developmental stimulation. Many mothers are still school-aged or very young, and may have limited insight into the importance of appropriate and adequate developmental care for their infants and the long-term implications thereof.
Sensory modulation and praxis are two important aspects of sensory integrative function that influence infant developent ${ }^{5}$. Sensory modulation profiles describe how infants register sensory input, orient or attend to it, and then interpret it. It reflects the infant's ability to manage behavioural reactions to sensation according to their sensory thresholds ${ }^{5}$. Praxis refers to the infant's ability to use sensory input as the foundation to conceptualise, organise and direct unfamiliar, purposeful motor actions and sequences ${ }^{6,7}$. It forms the bridge between cognition and motor abilities, as well as the final two steps of the sensory integration process 5 .

Infants (0 to 12 months) who experience sensory modulation difficulties may present with challenges in terms of (i) arousal - the infant may be unable to maintain alertness and make transitions between states of arousal; (ii) attention - the infant may be unable to focus selectively on a desired stimulus or task; (iii) affect - the infant may respond in emotionally inappropriate ways towards sensory stimuli; and (iv) action - the infant may be unable to engage in adaptive, goal-directed behaviour.

Balanced self-regulation is necessary to obtain a good fit between an infant's sensory integrative capabilities and the demands of the physical and social environments for optimal development and wellbeing 5 .

Infants with dyspraxia may have difficulties with one or more of the three interdependent steps of praxis, namely (i) ideation - the 
ability to formulate a goal for action based upon a perception of what is possible in the environment; (ii) motor planning - the ability to figure out specifically how to accomplish the goal through problem solving, sensorimotor awareness of the body and sequencing actions; and (iii) executing - carrying out the planned action, for example, an infant presented with an empty box may be unable to creatively explore the box ${ }^{5}$. Both sensory modulation profiles and dyspraxia are based on the infant's underlying capacity to process sensory input. The Infant/Toddler Sensory Profile (ITSP, 0-36 months) is a descriptive framework that provides insight into the sensory or motor dysfunction that underlies or contributes to worrisome behaviours ${ }^{5}$.

In terms of intervention within the NICU, neonatal physiologic stability and brain development are supported through light and sound modifications, therapeutic positioning, nurturing touch, non-nutritive sucking, alterations in caregiver timing and handling techniques, preservation of sleep and increased family involvement ${ }^{8}$. Mothers are encouraged in the NICU's to Kangaroo Mother Care their infants as much as possible since it holds numerous benefits for the infant, amongst others: better weight gain, encouraging earlier breast feeding and reducing the incidence of hospital acquired infections?.

A recent study by Nieder-Heitmann ${ }^{10}$, indicated that a sensory developmental care programme (SDCP) in the NICU benefitted the long term development of sensory functions in very low birth weight (VLBW) pre-term infants up to the age of 18 months corrected age.

Van Jaarsveld" adapted and presented a Model for Clinical Reasoning (MCR) on Possible Sensory Integration (SI) Difficulties and Dysfunctions, in an attempt to understand the different SI difficulties and dysfunctions ${ }^{\prime \prime}$. This model will be used to discuss the research results. Forming a comprehensive understanding of possible difficulties and dysfunctions based in SI functions is not an easy task. It is, however, evident that SI deficits affect an infant's normal development and emotional wellbeing.

Approximately $80 \%$ of the South African population are dependent on the public health services funded by the government $^{4}$. Regrettably, the public health system is under-resourced with over-crowded facilities and long waiting lists ${ }^{4}$. Due to budget constraints, the public health service is focused on the provision of basic healthcare and health services such as occupational therapy to prevent developmental delays or promote functional outcomes do not enjoy priority. Since premature infants' somatosensory processing deficits could affect their progress and performance until their school-aged years ${ }^{12}$, delays and difficulties place further financial burdens on the government, not only in terms of the Department of Health, but also the Department of Education. It is therefore necessary to determine the developmental status and prevalence of SI difficulties in premature infants to emphasise the importance of early SI intervention, not only for the $18 \%$ of the population that can afford private services ${ }^{4}$, but specifically for the majority of the population depending on government services.

The aim of this study was to identify the prevalence of sensory processing difficulties in premature infants from low socioeconomic backgrounds in a tertiary hospital in Bloemfontein, South Africa.

\section{METHODS}

\section{Study design}

A descriptive, observational study was conducted. Three standardised assessments (see Measurement tools) and a parent questionnaire were used to determine the prevalence of SI difficulties in a group of 24 premature infants.

\section{Population}

A tertiary hospital in Bloemfontein in the Free State Province of South Africa was chosen for the research since the hospital runs a large NICU and high-care unit. Twenty-four premature infants from the hospital's High Risk Infant Clinic with a birth weight of 750-1499 g [extremely low birth weight (ELBW) to very low birth weight (VLBW)], and a gestational age between 26 and 36 weeks (prematurity), with a corrected age [obtained through subtracting the number of weeks born premature from the infant's chronological age] of between 4 and 10 months, and who were medically stable, were included in this study. All infants adhering to the inclusion criteria and whose parents gave consent were included. All mothers of participating infants had implemented kangaroo mother care in the NICU as part of the hospital's developmental care programme.

Infants excluded from the study were those who had received any previous occupational therapy or SI intervention or, in addition to prematurity, had been diagnosed with any condition or neurological abnormalities that may have influenced typical development.

\section{Measurement tools}

The participating infants' sensory processing abilities and reactivity were measured with the, Infant/Toddler Sensory Profile (ITSP) ${ }^{13}$ and Test of Sensory functions in Infants (TSFI) ${ }^{14}$. The Bayley III Scales of Infant and Toddler Development (B-III) ${ }^{15}$ were used to observe the functional performance and developmental profile of the infants. Demographic and anthropometric data were gathered through a parent questionnaire compiled for the purposes of this research.

The ITSP (0-36 months) evaluates the possible contributions of sensory processing to the infant's daily performance patterns. It is a parent/caregiver questionnaire in which each item describes the infant's responses to various sensory experiences e.g. resistance to being cuddled or having hair washed according to the perception of the parents and therefore provides valuable perspectives on the infants' strengths and challenges in terms of behaviour. The areas of sensory processing investigated by this assessment tool are low registration, sensation seeking, sensory sensitivity, sensory avoiding and low threshold behaviour, as well as auditory, visual, tactile, vestibular and oral sensory processing ${ }^{13}$.

The TSFI (4-18 months) measures five subdomains of sensory processing and reactivity that have a strong impact on the development of SI in infants ${ }^{5}$. It can be used in the assessment of infants with developmental delays, regulatory disorders and those at risk for learning and sensory processing disorders, including highrisk premature infants. The TSFI is recommended to be used in conjunction with other developmental tests such as the Bayley III Scales of Infant and Toddler Development and other standardised assessments to provide an overall indicator of the infant's developmental functioning, in order to make decisions regarding the infant's developmental status ${ }^{14}$.

The B-III (I-42 months) assesses the developmental functioning of infants and toddlers across five domains, namely cognitive, language, motor (fine and gross motor), social-emotional and adaptive behaviour ${ }^{15}$.

The validity and reliability of all three assessment tools have been reported in the literature ${ }^{13-15}$. Although these tests were not developed specifically for the South African population, they were found to be most suitable for the purpose of this research.

\section{Method}

All the assessments were done on the same day for each infant and their corrected ages were used for the scoring and interpretation of results. The infants' parents were involved through the completion of the parent questionnaire and the ITSP caregiver questionnaire, with the assistance of the assessing therapist. During parent interviews, the assessment therapist completed the parent questionnaires by recording the answers provided by the parents onto the questionnaires. Parents could answer the questionnaire in English or Afrikaans and a translator was used if they were not proficient in one of these languages.

All participating infants were then formally assessed with the TSFI according to the five subdomains of sensory processing and reactivity, namely reactivity to tactile deep pressure, adaptive mo- 
tor functions, visual tactile integration, ocular-motor control and reactivity to vestibular stimulation.

The B-III was used to assess the developmental status of the infants. Assessment of the cognitive, language and motor domains was conducted using items administered to the infants. Assessment of the social-emotional and adaptive behaviour domains was conducted using the parents/primary caregivers' responses to a questionnaire.

One occupational therapist trained and experienced in administrating the three assessments conducted the tests on all 24 infants to eliminate concerns relating to inter-rater reliability. All tests were conducted at the hospital between $08 \mathrm{~h} 00$ and $\mathrm{I} 2 \mathrm{~h} 00$ in the morning with at least one parent or care giver present.

\section{Data analysis}

For the demographic data, various descriptive statistics are reported, including frequencies and percentages for categorical data, and means and standard deviations or medians and percentiles for continuous data. Where applicable; frequencies, means, and medians are also reported for the various scales and subscales.

\section{Ethical considerations}

Informed consent was obtained according to the General Guidelines of the University of the Free State Ethics Committee of the Faculty of Health Sciences (ECUFS NR II7/20II)16 ie from the Ethical Committee, and other relevant authorities, such as the Head: Clinical Services of the Hospital, Head of Department, Pediatrics and Child Health, University of the Free State and the Academic Hospital to conduct the study. Informed consent was obtained from the participating premature infants' parents. Parents were aware that they could withdraw their infants from participating in the study at any time without any negative consequences.

\section{RESULTS}

\section{Demographic data}

The demographic profile of the study participants included the geographic and socio-economic status, ages, marital status, level of education, home language and occupations of parents. All 24 participants resided in a low socio-economic area in Bloemfontein, South Africa. The majority of participants' home language was Sesotho $(n=17 ; 70.8 \%)$ and other languages included IsiXhosa, Setswana, Afrikaans and Bengali. The mean age of the infants' mothers and fathers was 28 years and 33 years respectively. Most $(n=17 ; 70.8 \%)$ were married or cohabiting and the remainder $(n=7 ; 28.2 \%)$ were single parents.

In terms of the parents' highest level of education, I 3 $(54.2 \%)$ of the mothers and $16(66.7 \%)$ of the fathers had matriculated, while two $(8.3 \%)$ of the mothers and three $(12.5 \%)$ of the fathers had attended college or work-related training. Only two (8.3\%) of the mothers had no schooling or completed grade 2 , while seven (29.2\%) of the infants' mothers and seven (29.2\%) of the fathers had completed grades eight to II. The majority of the mothers $(n=17 ; 70.8 \%)$ were unemployed, while working mothers were mostly unqualified or casual workers $(n=6 ; 25.0 \%)$. One $(4.2 \%)$ mother (4.2\%) had a formal qualification. The majority of fathers $(n=19 ; 79.2 \%)$ were unqualified or casual workers, four ( $16.7 \%$ ) fathers had formal qualifications, and one father was unemployed.

\section{Anthropometric data}

The anthropometric profile of the study population for the purpose of this study included the following: infants' gestational ages, corrected ages, genders and birth weights, as well as other relevant post-natal information, such as reasons for premature birth, selfsoothing methods used, implementation of kangaroo mother care in the NICU and the number of weeks hospitalised. As shown in Table I, I4 (58.3\%) of 24 participants were girls. According to
Table I: Gender and corrected ages of the study population

\begin{tabular}{|l|c|c|c|c|c|c|}
\hline \multirow{2}{*}{$\begin{array}{l}\text { Corrected } \\
\text { age group }\end{array}$} & \multicolumn{4}{|c|}{ Gender } & \multicolumn{2}{|c|}{ Total } \\
\cline { 2 - 7 } & \multicolumn{2}{|c|}{ Male (n = 10) } & \multicolumn{2}{|c|}{ Female (n= 14) } & \multicolumn{2}{|c|}{} \\
\cline { 2 - 7 } & $\mathbf{N}$ & $\%$ & $\mathbf{n}$ & $\%$ & $\mathbf{N}$ & $\%$ \\
\hline 4 - 6 months & 4 & 16.7 & 8 & 33.3 & 12 & 50.0 \\
\hline 7 - 9 months & 5 & 20.8 & 3 & 12.5 & 8 & 33.3 \\
\hline 10 - 12 months & 1 & 4.2 & 3 & 12.5 & 8 & 16.7 \\
\hline Total & 10 & 41.7 & 14 & 58.3 & 24 & 100 \\
\hline
\end{tabular}

their corrected ages, $12(50.0 \%)$ of the infants were in the 4-6 month age group.

The participating premature infants' corrected ages ranged from a minimum of 3 months and $I 7$ days to II months (Table II). The mean corrected age was 7 months. The infants' gestational age ranged between 28 weeks and 35 weeks, with a mean gestational

Table II: Premature infants' $(n=24)$ corrected and gestational ages and birth weight

\begin{tabular}{|l|c|c|c|c|}
\hline Variable & Minimum & Maximum & Mean & Median \\
\hline $\begin{array}{l}\text { Corrected age } \\
\text { (months: days) }\end{array}$ & $3: 17$ & $11: 0$ & $7: 0$ & $7: 0$ \\
\hline $\begin{array}{l}\text { Gestational age } \\
\text { (weeks) }\end{array}$ & 28 & 35 & 30.5 & 30 \\
\hline $\begin{array}{l}\text { Birth weight } \\
\text { (gram) }\end{array}$ & 750 & 1490 & 1150.6 & 1120 \\
\hline
\end{tabular}

age of 30.5 weeks. Their birth weight ranged from $750 \mathrm{~g}$ to $1490 \mathrm{~g}$, with a mean birth weight of II50.6g.

Preeclampsia (pregnancy-related hypertension with proteinuria and/or oedema) was the reason for premature birth in most of the infants $(n=17 ; 70.8 \%$. Other reasons for prematurity included hydramnios (excess amniotic fluid around the foetus) and the mother expecting twins or multiples. Infants were hospitalised for between three weeks and 12 weeks and the number of weeks mothers participated in kangaroo mother care ranged from one week to 36 weeks.

As shown in Figure $I$, finger sucking was used by more than half of infants $(n=13 ; 54.2 \%$ for self-soothing. Both finger and dummy sucking were used by six $(25.0 \%)$ of the infants. One infant was unable to self-soothe and the mother used breastfeeding as a method to calm her baby.

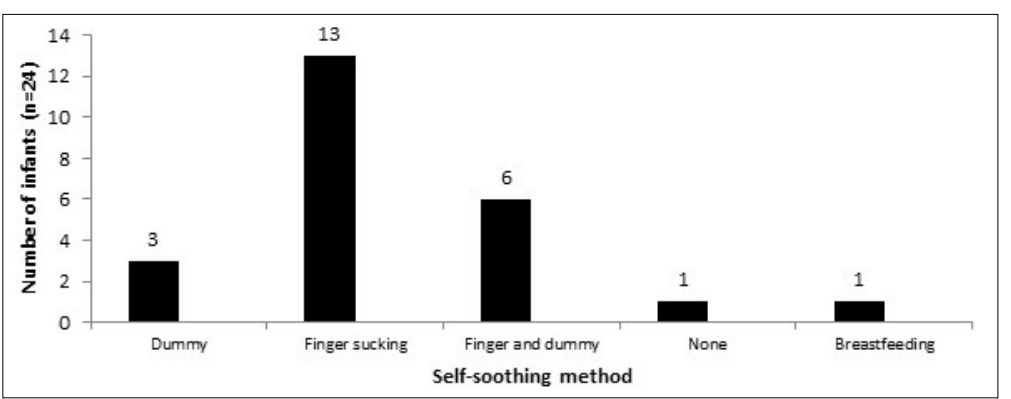

Figure I: Self-soothing methods used by premature infants

The results of the ITSP (Figure 2 on page 18) indicated that the majority of infants presented with difficulties in all the subtests except for sensation seeking behaviour, in which $66.7 \%$ infants fell within the typical performance range.

Low registration: It has been hypothesised that infants in the "more"- or "much-more"-than-others ranges have inadequate neural activation to support active participation ${ }^{6}$. They may miss 


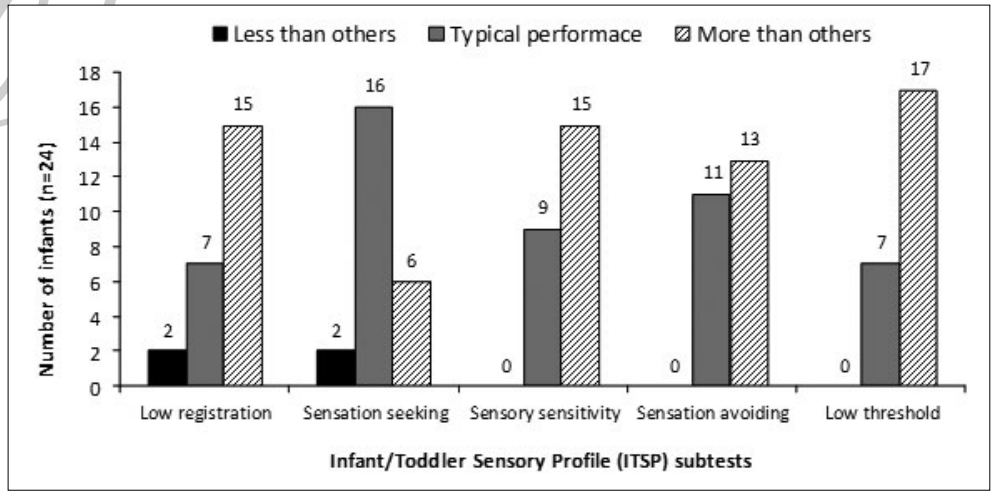

Figure 2: Results of the Infant-Toddler Sensory Profile (ITSP)

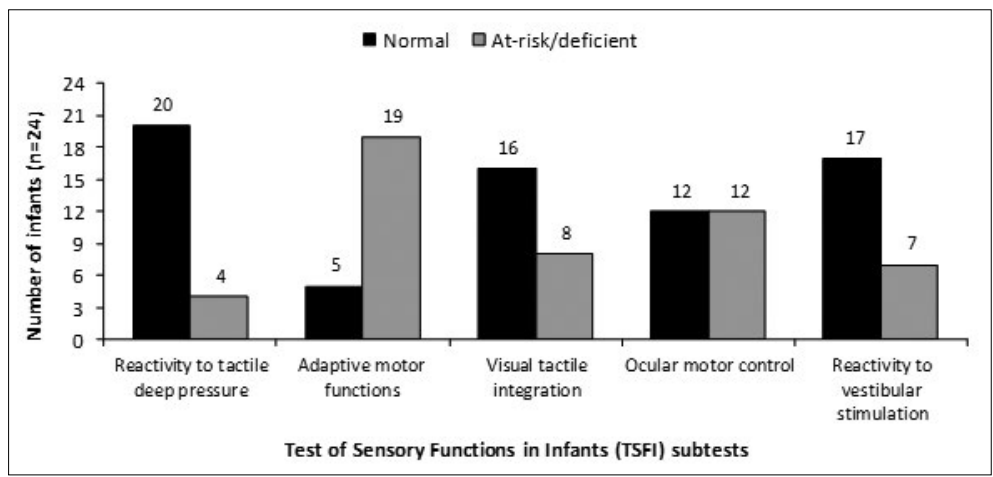

Figure 3: Results of the Test of Sensory Functions in Infants (TSFI)

noticeable cues to enable on-going engagement in activities ${ }^{13}$. A majority of $70.8 \%$ infants $(n=17)$ presented with behavioural response difficulties to all types of sensation experienced in their environment (for example, the infant being unaware of dirty diapers or not noticing when people enter the room ${ }^{6}$ ). Seven $(29.2 \%)$ of the infants presented with typical low registration behaviour.

Sensation seeking: This subtest measures infants' interest in and pleasure with all types of sensations, which may, for example, include actions such as enjoying looking at its own reflection in the mirror or enjoying rhythmical activities $^{\prime 3}$. Behaviour consistent with sensation seeking represents high neurological thresholds ${ }^{13}$. The results indicated that the majority of infants $(n=16 ; 66.7 \%)$ presented with typical sensation seeking behaviour. Six $(25.0 \%)$ infants fell in the more-than-others range and two $(8.3 \%)$ in the less-than-others range.

Sensory sensitivity: This subtest measures an infant's ability to notice and react to all types of sensation; for example, infants are distracted and/or have difficulty eating in noisy environments or become agitated when having their hair washed ${ }^{\prime 3}$. Behaviour consistent with sensory sensitivity represents low neurological thresholds and a tendency to react to sensory stimuli accordingly ${ }^{13}$. Fifteen infants $(62.5 \%)$ fell within the probable difference (more-than-others) range and nine (37.5\%) presented with typical sensory sensitivity behaviour.

Sensory avoiding: An infant's need for controlling the number and type of sensations experienced is measured through the items included in this subtest; for example, infants avoid getting their face/nose wiped or they try to escape from noisy environments ${ }^{13}$. Behaviour consistent with sensation avoidance represents low neurological thresholds with a tendency to actively work to keep these thresholds from being met. Sensation avoiders in the "more" or "much-more" than others ranges might engage in very disruptive behaviours. It has been hypothesised that meeting thresholds is uncomfortable or frightening to the infant. Infants react by either withdrawing or engaging in an emotional outburst that enables them to get out of the threatening situation ${ }^{13}$. The results indicated that $13(54.2 \%)$ infants presented with a probable difference (more-than-others), while II (45.8\%) infants fell within the typical performance range.

Low threshold: This subtest of the ITSP serves as a combined quadrant of the 'sensory sensitivity' and 'sensation avoiding' quadrants, and is relevant in terms of some aspects of poor sensory processing ${ }^{13}$. These infants' behaviour represents low neurological thresholds and they tend to be fussy and require a great deal of structure. This apparent inconsistency reflects the nervous system's attempts to simultaneously respond to stimuli and protect itself by reducing input, therefore producing variable responses ${ }^{13}$. The majority of infants $(n=17 ; 70.8 \%)$ fell within the probable difference range, and seven (29.2\%) presented with typical low threshold behaviour.

Results: Test of Sensory Functions in Infants (TSFI) The results of the TSFI (Figure 3) indicated that the majority of infants presented with normal scores regarding reactivity to tactile deep pressure $(n=20 ; 83.3 \%)$, visual tactile integration $(n=16 ; 66.7 \%)$ and reactivity to vestibular stimulation $(n=17 ; 70.8 \%)$. However, in terms of adaptive motor functions, 19 (79.2\%) infants presented with at-risk/ deficient results. On the ocular motor control subtest, the infants were evenly distributed for both normal and at-risk/ deficient scores.

\section{Results: Bayley III Scales of Infant and Toddler Development (B-III)}

The five main subtests of the B-III, namely cognitive, language (receptive and expressive), motor (fine and gross), social-emotional and adaptive behaviour, are characterised according to a certain level of performance. These levels are very superior, superior, high average, average, low average, borderline and extremely low ${ }^{4}$. The results of the B-III, shown in Figure 4, indicated that the participating infants' mean standard scores of all subtest ranged from average to low average.

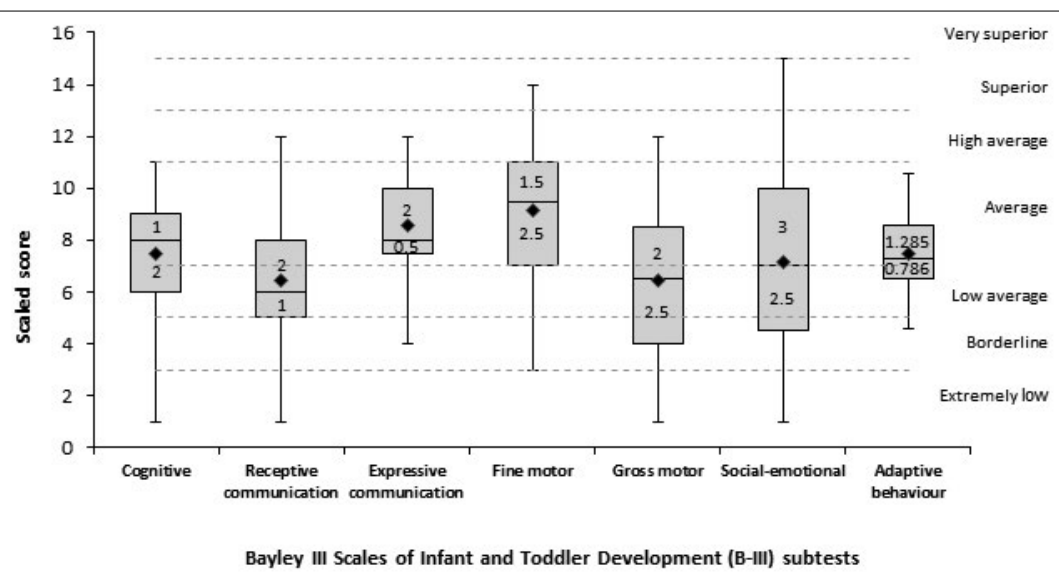

Figure 4: Results of the Bayley III Scales of Infant and Toddler Development (B-III)

Cognitive: The mean score for the cognitive subtest was 7.5 (low average). The third and fourth quartiles indicated that 12 (50.0\%) infants had standard scores between 8 and II, and fell in an average level of performance. Infants with standard scores from I to 8 were spread more widely and their level of performance ranged from extremely low to average.

Receptive communication: The mean score for the receptive communication subtest was 6.4 (within the low average band). Half $(50 \%)$ of the infants' standard scores ranged from I to 6 . Their performance levels ranged from extremely low to low average. 
Infants' results in the third and fourth quartiles were more widely spread and ranged from standard scores 6 to 12 (low average to high average).

Expressive communication: In terms of expressive communication, the infants' standard scores ranged from 4 to 12 , with a mean score of 8 (average). Half of the infants' performance levels ranged from borderline to low average while the other half of the infants' performance levels ranged from low average to high average.

Fine motor: The mean standard score for the fine motor subtest was 9.I (average). The third and fourth quartiles of the fine motor subtest indicated that twelve (50.0\%) infants had standard scores between 9 and 14 and fell in an average to superior level of performance. Infants with standard scores from 3 to 9 were more widely spread and their level of performance ranged from extremely low to average.

Gross motor: The infants' gross motor standard scores ranged from I to 12, with a mean score of 6.4 (low average). Half of the infants' performance levels ranged from extremely low to low average, while the other half's performance level ranged from low average to high average.

Social-emotional: The mean score for the social-emotional subtest was 7.2 (low average). Half of the infants' standard scores ranged from I to 7 . Their performance levels ranged from extremely low to low average. Infants' results in the third and fourth quartiles were more widely spread and had standard scores ranging from 7 to 15 (low average to superior).

Adaptive behaviour: In terms of the adaptive behaviour subtest, the infants' standard scores ranged from 4.6 to 10.6 , with a mean score of 7.5 (low average). Half of the infants' performance levels ranged from borderline to average, while the other $50 \%$ of infants' performance level ranged from low average to average.

\section{DISCUSSION}

The three stages of the MCR" " were used to form a comprehensive picture of the possible SI and developmental difficulties experienced by participating premature infants. Sensory registration can be seen as the first part of the process of modulation. Arousal and modulation enables an infant to sustain engagement despite variability. The second stage, discrimination and perception (spatial and temporal qualities of information), is viewed as discriminative use of sensory information. The final stage is where integration of sensory information contributes to skills and praxis (refined use)" ${ }^{\prime \prime}$.

The amount of sensory information needed for the brain to register is partially dependent on neurological thresholds and differs for each individual " . Behaviours consistent with low registration and sensation seeking represent high neurological thresholds, although a child with low sensory registration responds differently to sensory stimuli compared to a child who seeks sensory input ${ }^{13}$.

Sensation seeking behaviour is characterised by a developmental trend, with infants and toddlers 7 to 36 months of age engaging in more sensory seeking behaviours ${ }^{13}$. This creates a broader range of acceptable performance and children have a better chance of obtaining scores somewhere along this developmental continuum ${ }^{13}$. It correlates with our results of $66.7 \%(n=16)$ of infants who presented with typical sensation seeking behaviours falling in the acceptable performance range for infants in this age group. Literature indicated that compared to children without disabilities, only children with slight language delays had minor differences in sensory seeking behaviour ${ }^{13}$. The results of this study indicated that $33.3 \%(n=8)$ of infants presented with probable difference scores in terms of sensation seeking behaviour. These infants are driven to meet their high neurological thresholds and create opportunities to increase sensory input through active exploration and sensory play ${ }^{13}$. This behaviour is interpreted as beneficial for the development of premature infants who were initially deprived of sensory experiences, especially in terms of movement, tactile and proprioceptive input.
The majority of $70.8 \%(n=17)$ of infants presented with low registration behavioural responses to sensation, and these infants failed to notice stimuli more often. Infants with developmental delays and sensory integrative disorders could experience difficulties in terms of low registration behaviour, as well as have low thresholds for certain stimuli and tended to be more sensitive and/ or withdrawn from stimuli they noticed ${ }^{13}$.

In consideration of these results, it seems that premature infants could present with SI and developmental difficulties/disorders. They experience challenges of discriminating and perceiving the sensory information from their environment, thus influencing sensory modulation and praxis skills that are important contributors to development ${ }^{5}$.

The presence of low thresholds and low registration indicates poor modulation. These infants may not register stimuli of importance, but when they do their nervous systems have intolerance for input ${ }^{13}$. Without adequate modulation of sensory information, the infants are unable to sustain engagement in activities. Sensory modulation is also necessary for optimal levels of arousal to engage in activities, for stability in emotions and for behaviour ${ }^{\prime \prime}$.

Inadequate self-soothing methods used by the participating infants to assist them with sensory modulation may contribute further to poor discrimination of sensory stimulation. Although it is encouraging that $79.2 \%$ of the participating infants used finger sucking for self-soothing, a variety of techniques is recommended to enable the infant to engage in activities for exploration, play, interaction and to establish adequate sleep patterns. Only $37.5 \%$ $(n=9)$ infants used dummy sucking and $12.5 \%(n=3)$ had no other appropriate self-soothing methods. Dummy sucking enables infants to self-regulate while participating in exploration and play activities, while finger sucking or finger playing with a soft blanket or cloth in addition enables infants to self-regulate when tired or drowsy and they need to calm down for a good night's sleep.

Only once an infant is able to register and modulate sensory information adequately, will it be able discriminate sensory input. Sensory discrimination is about interpreting the qualities of the sensory information and adding meaning to it by forming perceptions ${ }^{\prime \prime}$. The infants have to use past experiences and memories from associations about the spatial and/or temporal qualities of what they experience and then act on that ${ }^{\prime \prime}$.

The majority of infants presented with normal reactivity to tactile deep pressure $(n=20 ; 83.3 \%)$, a test item designed to activate the tactile protective system ${ }^{5}$, visual tactile integration $(n=16$; $66.7 \%$ ), a test item designed to activate the tactile discriminative system ${ }^{14}$, and also reactivity to vestibular stimulation $(n=17$; $70.8 \%$ ), a test item that assesses infants' tolerance of movement in different planes in space (vertical, circular and inverted) $)^{14}$. The majority of the infants therefore were able to register, modulate and discriminate sensory information in terms of the three primary systems described and focussed on in Ayers Sensory Integration namely tactile, proprioceptive and vestibular systems.

The results of the adaptive motor functions in which $79.2 \%$ $(n=19)$ of the infants presented with at-risk/deficient scores were however a concern, since this is an indication of poor SI. These infants are not able to participate meaningfully and in reaction to sensory stimuli experienced through daily activities and occupations ${ }^{\prime \prime}$.

The results of the ocular motor subtest, where $50 \%(n=12)$ of the infants presented with at-risk/deficient ocular-motor control, is indicative of poor vestibular and proprioceptive processing ${ }^{17}$. An infant's eye and neck muscles play a very important role in organising the vestibular system. The ability to focus on objects while moving either the head or the object is an important developmental building block of early infancy. As a result, poor postural reactions can also be experienced during rolling over or getting into a creeping position, affecting the infant's foundation for standing and walking. These difficulties can contribute to further developmental challenges and can hinder the development of adequate motor and praxis abilities. Developmental outcomes in areas such as organised behaviour and motor actions can be affected negatively as a result ${ }^{17}$. 
Items evaluated on the B-III represent what Jean Ayers has termed "end products", which include the ability to concentrate, organise information, learn, think abstractly and reason, specialisation of each side of the body and the brain, self-esteem, self-control and self-confidence ${ }^{11,17}$.

The development of tactile discrimination plays an important role in adaptive motor behaviours, particularly in the initiation and planning of movement and exploration of the environment ${ }^{18,19}$. Tactile discrimination develops in conjunction with visual-spatial skills ${ }^{13}$. As the infant manipulates and explores his/her environment by touch, the visual-spatial properties of the object are encoded simultaneously ${ }^{13}$. Visual-tactile integration skills form the foundation for adaptive motor functions or early motor planning ${ }^{13}$.

The vestibular system plays an integral role in the development of body posture, muscle tone, ocular-motor control, reflex integration and equilibrium reactions ${ }^{17,20}$. Because the vestibular system is located in the junction of the brain halves where neural tracts from all parts of the brain converge for processing, it is also hypothesised that the vestibular system contributes to communication between the brain hemispheres and thus affects bilateral motor integration and hemispheric specialisation ${ }^{14,17}$. These vestibular-based functions have a strong impact on the development of motor skills, visual-spatial and language abilities, hand dominance and motor planning ${ }^{18,20}$. The normalisation of tactile and vestibular functions has been described as essential for the refinement of fine and gross motor skills and motor planning abilities ${ }^{14,17}$.

The mean standard scores on five out of seven subtests of the Bayley III Scales were found to be low average for cognitive, receptive communication, gross motor, social-emotional and adaptive behaviour subtests, with receptive communication and gross motor presenting with the lowest mean standard scores. Expressive communication and fine motor mean standard scores were average. These Bayley scores indicate that the majority of the participating infants experienced difficulties with the discrimination of tactile, proprioceptive and vestibular sensory input in terms of interpreting the quality of the input and adding meaning to it, and therefore affecting the infants' abilities to participate meaningfully and in a developmentally appropriate manner in daily activities.

\section{CONCLUSION}

The results indicated that premature infants can experience specific SI difficulties which affect their normal development within the first year. SI difficulties in terms of low thresholds, low registration of sensory input and modulation difficulties were identified, which in turn affect the infants' adaptive behaviour and ocular-motor control. The infants participating in this study experienced difficulties in all areas of development but specifically in terms of cognitive, receptive communication, gross motor, social-emotional and adaptive behaviour.

Infants and toddlers with poor SI typically exhibit delays in fine and gross motor skills, poor balance, incoordination and poor hand use. Distractibility, tactile defensiveness and problems with language and visual spatial skills may be apparent during the preschool years ${ }^{14}$. The identification of possible SI difficulties during infancy is therefore essential as a motivation for early SI intervention, in order to prevent escalating behavioural difficulties and developmental delays.

\section{ACKNOWLEDGEMENTS}

Dr. Daleen Struwig, medical writer, Faculty of Health Sciences, University of the Free State, for technical and editorial preparation of the manuscript; the study participants and their parents that made this study possible; the Sensory Integration Association of South Africa (SAISI) and the Research Committee of the School for Allied Health Professions of the University of the Free State for financial contributions towards this research project.

\section{REFERENCES}

I. Lawn J, Kerber K. Opportunities for Africa's newborns: Practical data, policy and programmatic support for newborn care in Africa.
Cape Town, PMNCH. 2006.

2. Lubbe W. Prematurity: Adjusting your dream. Pretoria: Little Steps, 2008.

3. Ayres AJ. Sensory Integration and the Child, Understanding Hidden Sensory Challenges. $25^{\text {th }}$ Anniversary ed. Los Angeles: Western Psychological Services, 2008.

4. Angloinfo the global expat network South Africa. Healthcare in South Africa http://southafrica.angloinfo.com/healthcare/health-system/ (accessed 25 February 2014).

5. Williamson GG, Anzalone ME. Sensory integration and self-regulation in infants and toddlers: Helping very young children interact with their environment. Zero to three. Washington DC: National Center for Infants, Toddlers and Families, 200I.

6. Ayers AJ. Developmental dyspraxia and adult onset apraxia. Torrance, CA: Sensory Integration International, 1985. Cited by Williamson GG, Anzalone ME. Sensory integration and self-regulation in infants and toddlers: Helping very young children interact with their environment. Zero to three. Washington D.C: National Centre for Infants, Toddlers and Families, 200I.

7. Cermak SA, Somatodyspraxia. In: Fischer AG, Murray EA, Bundy $A C$, editors. Sensory integration: Theory and practice. Philadelphia: Davies FA, 1991: 137-171. Cited by Williamson GG, Anzalone ME. Sensory integration and self-regulation in infants and toddlers: Helping very young children interact with their environment. Zero to three. Washington D.C: National Centre for Infants, Toddlers and Families, 200I

8. Hunter JG. Areas of pediatric occupational therapy services: neonatal intensive care unit. In: Case-Smith J, O'Brien J, editors. Occupational Therapy for Children. $6^{\text {th }}$ ed. Missouri: Elsevier Inc., 2010.

9. Faure F. Your Sensory Baby. Gentle routines for happy days and peaceful nights. London: Dorling Kindersley Ltd., $201 \mathrm{I}$.

10. Nieder-Heitmann E. The impact of a sensory developmental care programme for very low birth weight preterm infants in the neonatal intensive care unit. Cape Town: University of Stellenbosch, Tygerberg, 2010. http://scholar.sun.ac/za/handle/I0019.1/3180 (accessed 27 February 20II).

II. Van Jaarsveld A. Discussion on clinical reasoning on possible difficulties and dysfunctions. SAISI Newsletter, 20I I; 2 I (3): 7-I3.

12. DeMaio-Feldman D. Somatosensory processing abilities of very lowbirth weight infants at school age. American Journal of Occupational Therapy, 1994; 48(7): 643.

13. Dunn W. Infant Toddler Sensory Profile. San Antonio: The Psychological Corporation, 2002.

14. DeGangi GA, Greenspan SI. Test of Sensory Functions in Infants (TSFI) Manual. California: Western Psychological Services, 1993.

15. Bayley N. Bayley Scales of Infant and Toddler Development. Administration Manual. $3^{\text {rd }}$ ed. San Antonio: Harcourt Assessment, Inc., 2006.

16. University of the Free State: Faculty of Health Services. General guidelines of the University of the Free State Ethics Committee. http:// health.ufs.ac.za/content.aspx?uid = 13 (accessed 25 February 2014).

17. Ayres AJ. Sensory integration and the child: Understanding hidden sensory challenges. $25^{\text {th }}$ Anniversary ed. Los Angeles: Western Psychological Services, 2008.

18. Ayers AJ. Sensory integration and learning disorders. Los Angeles: Western Psychological Services, 1972. Cited by DeGangi GA, Greenspan SI. Test of Sensory Functions in Infants (TSFI) Manual. California: Western Psychological Services, 1993.

19. Lederman SJ. Tactile perception and texture. In: Carterette E, Friedman M, editors. Handbook of Perception. New York: Alcot Publishers, 1973. Cited by DeGangi GA, Greenspan SI. Test of Sensory Functions in Infants (TSFI) Manual. California: Western Psychological Services, 1993.

20. Clark DL. The vestibular system: An overview of structure and function. Physical and occupational therapy. Pediatrics 1985; 5: 5-32. Cited by DeGangi GA, Greenspan SI. Test of Sensory Functions in Infants (TSFI) Manual. California: Western Psychological Services, 1993.

\section{Corresponding author}

Elise Lecuona

elise.lecuona@gmail.com 